\title{
Endoscopic Postoperative Recurrence Rates in Crohn's Disease in Korea: The Beginning of a New Approach?
}

\author{
Paulo Gustavo Kotze, Rogerio Saad-Hossne ${ }^{1}$, Antonino Spinelli ${ }^{2}$ \\ Colorectal Surgery Unit, Cajuru University Hospital, Catholic University of Paraná, Curitiba, PR, Brazil, Digestive Surgery Unit, University of \\ the State of São Paulo (UNESP) $)^{1}$ Botucatu, SP, Brazil, Colorectal Surgery Unit, Instituto Clinico Humanitas', Milano, Italy
}

\section{Dear sir,}

It was with great interest that we read the article entitled "Clinical and Endoscopic Recurrence after Surgical Resection in Patients with Crohn's Disease", recently published in this journal by Yang Woon Lee and colleagues. ${ }^{1}$ The article describes one of the first retrospective experiences with Korean patients regarding the rates of both clinical and endoscopic recurrence (ER) after surgical abdominal procedures for CD. In this study, the authors found an overall rate of ER of $71.4 \%$ ( 15 from 21) patients, in a mean follow-up period of 50.5 months.

Although the study adds very important real world data and represents one of the first experiences on the topic of ER in Korean patients, some considerations are important in this data analysis and must be taken into consideration.

First, the population of the study is somehow heterogeneous. A myriad of operations was performed as they fulfilled the inclusion criteria. Most of the western studies were performed only after ileocecal resections, ${ }^{2,3}$ and in this interesting Korean retrospective study, small bowel resections, segmental colectomies and other different procedures were also included. The Rutgeerts's score is not fully validated after different procedures, it is mostly widely used after ileocecal resections, and there is significant controversy if it can be applied after other operations, even with capsule endoscopy to detect recurrence. ${ }^{4}$ Moreover, most of the western studies consider the definition of ER as a Rutgeerts' score $\geq i-2$, con-

Received May 10, 2014. Revised May 10, 2014. Accepted May 10, 2014. Correspondence to Paulo Gustavo Kotze, Rua Bruno Filgueira, 369 - cj. 1205, CEP 80240-220, Curitiba, PR, Brazil. Tel: +55-41-3243-0033, Fax: +55-41-3243-0033,E-mail: pgkotze@hotmail.com

Financial support: None. Conflict of interest: None. sidering the i-1 score as mucosal normality, differently of the criteria of the Korean study. ${ }^{1-4}$ However, only one patient in the sample presented as an i- 1 after surgery, what would not influence significantly the final results of recurrence.

There is quite a consensus in the literature that smoking, previous resections and penetrating $\mathrm{CD}$ are significant risk factors for ER in CD patients. ${ }^{3,5}$ The study did not find any risk factor, despite meticulous statistical analysis, maybe for 2 reasons: the small number of patients in the sample, or the possibility of a different genetic background in Korean CD patients, that can alter the phenotype of the disease in the country, as previously stated by the authors.

We recently presented at the European Crohn's and Colitis Organisation (ECCO) congress the results from a database with patients from 7 referral centers from South America, Europe and Asia regarding the same topic as this interesting Korean study. ${ }^{6}$ In our experience, ER was observed in aproxi-

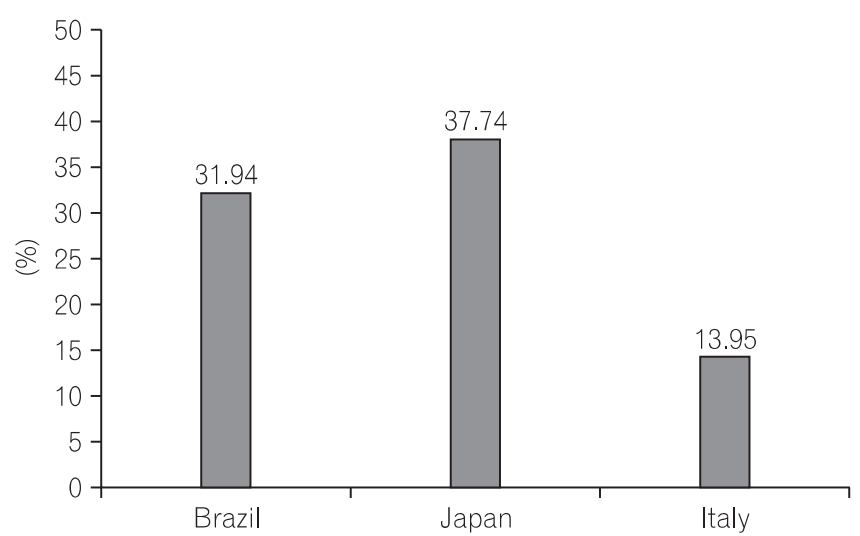

Fig. 1. Early postoperative endoscopic recurrence (PER). Early PER rates from the observational MULTIPER database, with data compiled from 3 different countries.

(c) Copyright 2014. Korean Association for the Study of Intestinal Diseases. All rights reserved.

This is an Open Access article distributed under the terms of the Creative Commons Attribution Non-Commercial License (http://creativecommons.org/licenses/by-nc/3.0) which permits unrestricted non-commercial use, distribution, and reproduction in any medium, provided the original work is properly cited. 
mately $32 \%$ of patients treated in Brazil, $38 \%$ of those treated in Japan and in $14 \%$ of patients treated in Italy (Fig. 1). These lower numbers, as compared to the Korean study, probably were observed due to the shorter follow-up period of 12 months in this database. However, they could also be a consequence of a similar strategy of postoperative prevention of recurrence that is actively practised in these referral centers. ${ }^{6}$ The differences between the Korean study as compared to ours, are also basically related to the different populations analyzed.

Despite these few considerations, we would like to congratulate the staff from the Catholic University of Korea for this new interesting information on ER after CD surgical procedures in Asian patients. We also hope that this data can impact in a near future in the prospective management of surgically-treated CD patients in Korea, increasing the number of colonoscopies or other endoscopic examinations performed 6 to 12 months after surgery, in order to detect early ER, optimize medical therapy if needed, and possibly to lead to a lower rate of recurrence in the long term and better control of the disease.

\section{REFERENCES}

1. Lee YW, Lee KM, Chung WC, Paik CN, Sung HJ, Oh YS. Clinical and endoscopic recurrence after surgical resection in patients with Crohn's disease. Intest Res 2014;12:117-123.

2. Spinelli A, Sacchi M, Fiorino G, Danese S, Montorsi M. Risk of postoperative recurrence and postoperative management of Crohn's disease. World J Gastroenterol 2011;17:3213-3219.

3. De Cruz P, Kamm MA, Prideaux L, Allen PB, Desmond PV. Postoperative recurrent luminal Crohn's disease: a systematic review. Inflamm Bowel Dis 2012;18:758-777.

4. Rutgeerts P, Geboes K, Vantrappen G, Beyls J, Kerremans R, Hiele M. Predictability of the postoperative course of Crohn's disease. Gastroenterology 1990;99:956-963.

5. Yamamoto T. Factors affecting recurrence after surgery for Crohn's disease. World J Gastroenterol 2005;11:3971-3979.

6. Kotze PG, Yamamoto T, Danese S, et al. P511 Does the location of an IBD centre impact the rates of early postoperative endoscopic recurrence after ileocecal resection in Crohn's disease? Results from the MULTIPER database. J Crohn's Colitis 2014;8:S281. 